\title{
Les associations thématiques du motif de l'eau dans un roman québécois : HKPQ de Michèle Plomer
}

\author{
Sánchez-Hernández, Ángeles
}

Universidad de Las Palmas de Gran Canaria, angeles.sanchez@ulpgc.es

\begin{abstract}
Resumen
Este estudio analiza el motivo del agua como símbolo de mediación cultural en la novela francófona. En primer lugar, revisamos los estudios teóricos que tratan la problemática simbólica y la interpretación del agua en la literatura para pasar posteriormente a aplicar estas teorías sobre un texto. Hemos elegido la novela HKPQ (2009) de la escritora canadiense Michèle Plomer. Las imágenes acuáticas trasladan los intercambios entre dos países (Canadá y China) comunicando igualmente el pasado con el futuro de la vida de la narradora. El agua se carga de una doble significación ya que soporta los temas de la regeneración y la metamorfosis que se operan en el espíritu y en cuerpo de la protagonista.
\end{abstract}

Palabras clave : crítica temática ; novela quebequense ; interculturalidad.

\section{Résumé}

Cette étude analyse le motif de l'eau comme symbole de médiation culturelle dans le roman francophone. D’abord, nous révisons la problématique symbolique et l'interprétation de l'eau dans les études théoriques, pour passer ensuite à les appliquer sur un texte. Nous avons choisi le roman HKPQ (2009) de l'écrivaine canadienne Michèle Plomer. Les images aquatiques véhiculent des échanges entre deux pays (le Canada et la Chine) et installent des passerelles du passé à l'avenir dans la vie de la narratrice. L'eau apparaît chargée d'une signification double véhiculant les thèmes de la régénération et de la métamorphose qui s'opèrent chez l'esprit et le corps du personnage principal.

Mots-clés : critique thématique ; roman québécois ; interculturalité.

\begin{abstract}
This study analyses the reason for water being a symbol of cultural mediation in the Francophone novel. First of all, we take a look at the theoretical studies dealing with the matter of symbols and the interpretation of water in literature, to then apply these theories to a text. We have selected the novel entitled HKPQ (2009) by the Canadian writer Michèle Plomer. It is the images of water that are the vehicles for the exchanges between the two countries (Canada and China), setting up a link between the past and the future life of the narrator. Water takes on a double meaning as it is the basis of the themes of regeneration and metamorphosis which take place in the spirit and in the body of the leading character.
\end{abstract}

Keywords : thematic criticism ; Quebecois novel ; interculturality. 


\section{Introduction}

L'eau établit l'axe d'étude de cette recherche. Elle est l'un des quatre éléments indispensable décrits par Sénèque dans Naturales Quaestiones et dont les interprétations se sont multipliées dans plusieurs disciplines. Le texte que nous avons choisi d'analyser dans cette communication, c'est le roman de Michèle Plomer : HKPQ (2009). L'œuvre fut récompensée par le prix littéraire 'France-Québec' en 2010. Notre but est de montrer comment le motif de l'eau intervient dans la narration, enrichissant l'univers imaginaire de nouvelles nuances et amplifiant une thématique déjà singulière, celle de la quête identitaire dans 'l'entre-deux'.

L'écrivaine canadienne Michèle Plomer est selon le magazine L'Actualité l'une des « 5 nouvelles voix qui secouent le roman québécois » ${ }^{1}$. Elle est l'auteure de plusieurs romans parus chez 'Marchand de feuilles'; son premier roman $L e$ Jardin sablier a été publié en 2007, ensuite HKPQ en 2009 et, de 2011 à 2013, elle a poursuivi sa production avec la trilogie sino-québécoise de Dragonville (Porcelaine, Encre et Empois). Elle partage aujourd'hui son temps entre les Cantons-de-l'Est au Québec et la Chine du Sud, deux géographies qui nourrissent son imaginaire et son écriture. Les sigles du titre font allusion à la ville de Hong-Kong où l'histoire se déroule et les deux dernières lettres PQ correspondent, au nom de la narratrice qui signe ainsi l'avant- propos du livre et dont la première lettre relie, en plus, au nom même de l'auteur Plomer ; et puis, la deuxième lettre renvoie au Québec, son pays d'origine.

Par conséquent, avec ces quatre lettres qui conforment le titre, on pourrait dire que le récit tient de l'autobiographie ou de l'autofiction car l'auteure laisse la trace indélébile d'un 'je' personnel en lien avec l'écrivaine. La narratrice de HKPQ est une jeune femme qui vient de quitter hâtivement son pays, le Québec, pour s'engager professionnellement en Chine. Dans cet autre pays, elle va faire des rencontres décisives : celle de Wang Xia, une jeune voleuse qui confie à la narratrice une lettre destinée à sa mère introuvable. Et puis celle d'un poisson femelle qui deviendra le centre d'une enquête et qui va accompagner cette femme vers sa guérison émotionnelle.

Le récit est guidé dès le début par des images en rapport étroit à l'eau : la raison du départ est liée au suicide de son fiancé noyé dans le (fleuve) Saint-Laurent, ensuite la rencontre de la jeune chinoise un jour de pluie et enfin la retrouvaille de Poissonne, belle comme un 'camée'. Nous allons exposer d'abord les études littéraires à propos des significations de l'eau dans l'imaginaire collectif pour passer ensuite à leur application au roman HKPQ de Michèle Plomer en essayant de démontrer quelle est l'histoire sous-jacente dans ce roman présenté comme une enquête policière.

\section{La problématique symbolique et l'interprétation de l'eau dans les études théoriques}

Dans le titre de cette communication nous avons employé les termes thème et motif. Ces deux notions essentielles sont souvent définies de façon hétérogène par les chercheurs des études thématiques appartenant à des courants divers et dont la terminologie est parfois contradictoire. Pour simplifier cette diversité, nous avons adopté la terminologie employée par Mattias Aronssons (2008 : 2-30) car elle nous semble la plus simple et adéquate. Nous allons donc utiliser le mot motif pour désigner les éléments (objets ou phénomènes) présents dans l’univers diégétique, tandis que le mot thème sera le résultat de notre interprétation.

Ce qu'on nomme 'motif' dans les études thématiques est très proche du terme 'symbole', élément qui constitue le noyau de toute activité symbolique consistant «à projeter sur un objet, un geste, ou une situation le contenu d'un affect renvoyant à un objet, à un geste, ou à une situation antérieure refoulée qui prend valeur fondatrice » (Dubois, 1983: 9). D’après Claude Dubois (1983: 11-13), le sujet se donne le moyen d'atteindre le réel par isomorphisme à travers l'imaginaire. C'est-à-dire, on établit une dynamique de 'miroir' ou de reflet, créant un mécanisme par lequel l'imaginaire se projette sur son image. Le sujet fait intervenir l'identité et l'altérité (puisque mon image est moi, je deviens autre) et on bâtit une illusion narcissique. Le symbole permet à l'écrivain de jouer sur les deux réseaux de sens : l'opposition psychique « conscient/inconscient» se répercute dans le langage en opposition «manifeste/latent».

L'imagination est l'expression d'une liberté capable d'établir des liens entre la conscience et le monde extérieur (Saxe Fernández, 1979 : 77). Les anciennes cosmogonies avaient fourni à Bachelard un critère de distinction entre

\footnotetext{
${ }^{1}$ Site Internet de l'auteure.
} 
les quatre types d'imagination matérielle, chaque type d'imagination se rattachant à l'un des éléments de manière privilégiée : terre, eau, air ou feu. Pour ce philosophe passionné de poésie, les vrais symboles sont les symboles qu’il appelle matériels car ils partent du monde pour nous parler, à la fois, de ce monde. Ces éléments servent à expliquer les couches profondes de l’imaginaire humain et le rapport de notre volonté avec le monde (Tarot, 2007 : 67). Bachelard (1942 : 5) avançait l’idée que les éléments matériels constituent des principes de première importance pour la création littéraire dans l'élaboration des « rêveries ».

Ces images matérielles qui structurent nos rêves sont l'expression des valeurs psychiques. Il montre que, si l'eau violente est une manifestation de la colère, il existe bien de manière des manières de la rêver. Et cette variabilité des rêves est particulièrement riche en littérature. Pour qu'une rêverie se poursuive avec assez de constance pour donner une œuvre écrite, il faut qu'elle trouve sa matière, il faut qu'un élément matériel lui donne sa propre substance, sa propre règle, sa poétique spécifique. Ainsi, selon Bachelard, l'eau n’est pas un motif quelconque, puisque c'est un des éléments susceptibles d'inspirer les poètes afin qu'ils modifient une "rêverie » en une œuvre littéraire. Il signale des auteurs pour qui l'eau est l'élément dominant ; et, en particulier, il signale Edgar Allen Poe pour qui l'eau est «la matière fondamentale pour l'inconscient » (Bachelard, 1942 : 87). Pour l'auteur américain, l'eau est associée à la pulsion de la mort car il avait été frappé à l’âge de deux ans par la mort de sa mère.

Des dictionnaires spécifiques essaient d’inventorier la portée des symboles dans l'imaginaire collectif. D’après le Dictionnaire psychanalytique des images et du symbole des rêves ${ }^{2}$ l'eau est l'élément le plus présent dans nos rêves recouvrant plusieurs formes et possédant une valeur ambivalente. Dans certains dictionnaires des symboles, comme ceux de Chevalier et Gheerbant (1995 : 52-56) ou de Nadia Julien (1989 : 115) cet élément se présente sous une double signification. D’une part, l'eau apparaît sous trois formes dominantes : comme source de vie, comme moyen de purification et comme centre de régénération corporelle et spirituelle. Les rêves d'eau gardent un sens régénérateur d'où surgissent toutes les formes de vie ; les bains dévoilent le besoin de l'être humain de se rénover et d’oublier le passé. L'eau claire et limpide montre le moment où le rêveur voit nettement en lui. L'eau est une source de purification dans bien des cultures. Elle semble caractérisée par une valeur nourricière, épuratrice et elle est aussi capable de métamorphose car elle prend la forme du réceptacle qui la contient.

Et d'une autre part, l'eau peut aussi engloutir et ravager étant donné qu'elle engendre parfois une force maudite. Si cette eau est trouble ou grise, cela signifie que l'individu se rend compte que son inconscient est encombré de choses refoulées. Si elle est noire, c'est un signe de totale rupture avec ce monde informel de l'inconscient. Le sujet doit surmonter sa peur et oser regarder à l’intérieur de soi car la reconnaissance de la partie obscure de soi est nécessaire à l'épanouissement personnel. Elle est susceptible d'être destructrice car l'eau renvoie souvent à l'invisible soit aux fonds obscurs soit à l'horizon invisible ; d’ailleurs, elle apparaît sous l'aspect de la menace cachée derrière un masque calme.

Dans la religion chrétienne, le baptême est signe de régénération. Le symbolisme du poisson est inséparable de celui de l'eau car il évoque le renouvellement universel de la nature dans la plupart des pays. Les Chrétiens ont fait du poisson, cet habitant des eaux, le symbole du sacrement du baptême, donc symbole de la naissance religieuse et spirituelle. En Chine, le poisson est associé à la richesse, à la vie et à la fécondité « en raison de son extraordinaire faculté à se reproduire » (Julien, 1989 : 311). Dans d’autres cultures orientales comme la khmère, les poissons se trouvent aussi dans les inscriptions à la base des monuments (Chevalier et Gheerbant, 1995 : 823), cette situation dans la partie la plus proche de la terre est due à son rapport aux eaux inférieures du monde submergé qui est aussi relié à la naissance ou à la restauration cyclique. En somme, l'eau est un élément ambigu comme le signale aussi Mircea Eliade (Goguey et Dubouchet, 2014 : 44 ; Bouguerra, 2007 :10). Dans ce monde du XXIe siècle de plus en plus multiculturel où les limites géographiques ne sont plus la marque indéfectible d’identité et d’appartenance culturelle, la sémiologie de l'eau possède un intérêt particulier car, il nous faut des référents communs à l’imaginaire collectif pour relier les cultures. Les deux 'motifs', l'eau et le poisson, seront l'objet d'étude dans ce roman québécois pour tenter de dégager les thèmes fondamentaux sur lesquels évolue le récit de Michèle Plomer.

\footnotetext{
${ }^{2}$ Site Internet sans foliotage.
} 


\section{Le récit de $H K P Q$ transporté par les symboles de l'eau}

Michèle Plomer, dans l'avant-propos de ce roman, explique que son livre est un carrefour où l'on trouve une hybridité des langues et des cultures. Se plongeant dans ce métissage, la narratrice essaye de recomposer son identité car elle avait été anéantie par sa dernière liaison amoureuse. Le départ de Québec s'était produit dans une situation d'angoisse profonde car elle y étouffait et, même, elle en mourait. Dans la préface, l'auteure insiste sur l'existence d'autres éléments qu'elle n'arrive pas à définir, tant il est difficile pour elle de les identifier. Elle nous parle de son désir de raconter son histoire "pendant qu'elle était fraîche dans ma mémoire et parce que les questions qu'on nous pose au retour de l'étranger ne sont pas toujours celles auxquelles on aurait envie de répondre. Et parfois on ne sait pas comment parler de l'essentiel » (Plomer, 2009: $7^{3}$ ). Cette impuissance à parler des sujets 'essentiels' est dépassée par un double niveau narratif, d'un côté celui de l'évolution de l'histoire qui avance comme une enquête policière et d'un autre côté un niveau sous-jacent où la poétique de l'eau développe une histoire plus intime et refoulée que le lecteur perçoit plutôt au niveau sensoriel. La narration révèle, à travers les images aquatiques, ce qu'elle était incapable de dire auparavant car la personnalité de H. -seule lettre pour désigner son conjoint-, était clairement manipulatrice. Face à cette domination masculine, elle se sentait impuissante et ne trouvait d'autre façon de s'enfuir qu'à travers sa propre disparition corporelle qui l'avait conduite à l'anorexie, seule manière possible de refuser son corps à son compagnon.

La narratrice quitte subitement le Québec après le suicide de son fiancé dans ce «long fleuve glacial » (p. 10) de Montréal. L'eau est ainsi perçue dans son sens objectif mais, en plus, comme miroir de la froideur amoureuse à laquelle elle ne savait pas mettre fin. Ensuite elle affirme être " partie pour cacher son soulagement » (p. 27); cet apaisement par la disparition de son partenaire devait être caché à la société et, pour ce faire, elle part pour la Chine où elle veut trouver une nouvelle chance pour reconstruire son 'je' physique et psychique se libérant des rapports maladifs. Le motif de l'eau sert à véhiculer avec naturel les échanges entre les deux pays -le Canada et la Chine-, établissant des passerelles du passé à l'avenir dans la vie de la narratrice. Les différentes manifestations de l'élément 'eau' vont se poursuivre et se transformer tout au long de la narration pour diriger ce je au féminin -toujours sans prénom- vers son salut.

La narratrice se noie dans le travail à son arrivée en Chine pour tenter de s'évader de la problématique personnelle. L'histoire évolue donc de l'image de la noyade figurée de la narratrice dans le travail au premier chapitre (s'appuyant quelques pages après sur la tragédie intime) en passant par le second chapitre où elle avoue la raison de sa fuite précipitée : la libération éprouvée par cette mort brutale, soulagement qu'elle ne désire pas étaler au regard de la société. On apprend donc assez tôt l'histoire qui la tracasse car elle se sent coupable du sentiment de liberté provoqué par la disparition de la figure tyrannique du rapport amoureux.

Le Saint-Laurent à Montréal construit le décor où se projette un autre fleuve chinois, celui de la deuxième histoire de l'intrigue, celle de la jeune voleuse Wang Xia rencontrée dans le train et qui lui confie une lettre pour sa mère : « J'étais libre. Comme Wang Xia, un fleuve m'avait affranchie » (p. 27). Le motif de l'eau se revêt d'abord du côté obscur et refoulé des rêves par les motifs du fleuve et de la noyade pour se relier ensuite à celui de la chance dans la culture chinoise grâce à Poissonne qui devient un personnage à part entière dans le roman. Elle sera à l'origine du changement existentielle de cette femme et avec qui elle entre "dans la lumière » (p. 21) et lui donnera la force de rentre au Québec en se disant «que la chance s'était fixée à moi » (p. 225). Cette rencontre lumineuse s’offre en parallèle à la confession des vraies raisons de son départ :

J'étais dans ma tête. Le cerveau engourdi par le film qui y était à l'affiche depuis quelques semaines : celui des événements qui m’avaient menée ici. Il m’était difficile d'expliquer, de traduire en mots convenables pour ceux qui s’inquiétaient de moi, pourquoi j'avais quitté le Québec de façon si subite. Mon entourage trouvait normal que j'aie besoin d'air frais après le saut de H., mais le non- renouvellement de mon contrat de travail à l'université leur semblait irréfléchi. [...] ils ont trouvé le corps défait de H. coincé entre deux roches sur une rive du Saint-Laurent (p. 25).

\footnotetext{
${ }^{3}$ A partir d’ici les références au roman de M. Plomer vont apparaitre avec le numéro de page seulement.
} 
Cette personnalité féminine meurtrie évolue à travers l'histoire que sous-tend une particulière métaphore filée appuyée sur le champ sémantique de l'eau et qui s'enrichit de celui du poisson, symbole pour l'écrivaine d'égalité car « le rapport de maître et de conquis était impossible entre l’humain et le poisson. Je savourais cette égalité. J’avais toujours occupé mon espace avec culpabilité » (p. 122). L’observation de cet être aquatique par lequel la narratrice retrouve une ‘compagne’ de vie lui fait réfléchir à l'existence des rapports humains vécus en égalité.

La fin du périple chinois va être marquée par le retour de la confiance de la narratrice en la nature humaine et en l'amitié. Elle a retrouvé une figure masculine protectrice qui lui a offert un modèle de comportement masculin qu'elle admire. Son collègue Liu Ju Lin. La narratrice affirme dans les dernières pages: " Mes pensées se sont réchauffées sous le jet de la douche. [...] Oui je désirais me rattacher à cela. J’avais foi en son amitié. Il était Chinois, le sens du devoir envers une amie était profondément inscrit en lui » (p. 220). L'eau accompagne une autre fois ce sentiment de régénération au moment où elle se sent traquée par la police, suspecte d'avoir volé cette particulière pièce d'aquarium, son collègue chinois imagine toute une histoire pour la tirer d'affaire et les sensations de chaleur se véhiculent encore par les images de l'eau qui coule sur son corps, elle se sent sauvée par cette amitié.

En revanche, le départ du pays canadien s'était passé dans des conditions troubles marquées par la détresse dans sa relation amoureuse qui l'avait rendue malade et incapable de s'en sortir. Son anorexie restait la seule manière de maîtriser son destin qu'elle avait trouvée pour affronter le tempérament tourmenté de son amoureux H. et elle affirme :

Je n'avais pas reconnu le trouble que le désaxait. A la fin de nous, [...] malgré son besoin de me dominer et de me blesser, j'aurais cru qu'une certaine pudeur [...] l'aurait empêché de faire revivre à sa famille la douleur d'une autre mort violente. [...] Il ne pouvait plus me marquer physiquement. Mon anorexie lui avait volé cet avantage. Mon être avait évacué la demeure et opérait dans un espace parallèle. Je gérais mon corps, je le maintenais en vie [...] mais je ne l'habitais plus (p. 156).

Elle était incapable de se soustraire à lui d'aucune autre façon, cependant elle était consciente de son envie d'oublier la folie dans laquelle ils vivaient. Au moment où elle apprend la mort de H., elle est libérée physiquement de sa présence car elle était attrapée par la peur qui l’empêchait de se séparer de lui à cause de sa brutalité. La même violence qu'il avait lui-même éprouvé dans son enfance avec son père. Dans la personnalité de ce jeune homme son désespoir et son mal de vivre s’imbriquaient «comme des poupées russes avec la peur de l’inconnu » (p. 151), H. suivra le même destin que son père, le suicide. Mais la jeune femme, lors de la disparition de son conjoint, se dit: “S’il s’était léché les babines à l’idée que je pâtisse de regrets, il m’avait mal jaugée » (p. 158). Donc elle prend la première offre qui surgit pour s'éloigner et recommencer sa vie loin de ces comportements déprimés et déprimants.

L'amélioration de la narratrice va de pair avec celle de Poissonne, toutes les deux logeaient dans un petit habitacle dans une ambiance calme et au milieu d'une atmosphère suintante d'humidité et de chaleur. Elle y retrouve peu à peu « un bien-être nouveau [qui] irradiait de [ses] cellules» (p. 69). Le personnage de Poissonne est une symbiose complexe car «elle n’était ni poisson, ni baleine, ni femme, mais un amalgame indéfinie des créatures » (p. 38). Elle est sans doute revêtue des caractères bien humains comme les mains. La narratrice est attirée par cet animal poussée par un sentiment d'engagement envers elle, elle s’octroie l'obligation de la tirer de « la noirceur et de la crasse » (p. 39) dans laquelle elle se trouvait ; c'est un miroir où elle se voit clairement qui lui rappelle le but du voyage en Chine : «je suis venue en Chine pour être humaine. Pour baigner dans le grand pool. Poissonne me rappelait à cela » (p. 50). À partir de là, cette jeune femme et ce poisson-femelle vont poursuivre un parcours ensemble qui sera favorable à la narratrice. Les perceptions qu'elle décrit à partir de l'observation du poisson seront les manifestations de ses états d’âme.

Nous avons déjà souligné dans le corps théorique de l'étude comment le sujet se donne le moyen d'atteindre le réel par isomorphisme à travers l’imaginaire. Plomer établit cette dynamique de 'miroir' pour créer ce mécanisme par lequel ces sentiments refoulés et difficiles à exprimer se projettent sur l'image de cet être de nature aquatique. La narratrice fait donc intervenir l’identité et l'altérité -puisque mon image est moi, je deviens autre- bâtissant ainsi une 
illusion narcissique. Et, à travers l'évolution du personnage de Poissonne, la partie inconsciente de cette identité blessée s'exprime par ce langage en opposition entre manifeste, l’histoire du récit, et latent, les sentiments ou émotions qui se expriment au travers de Poissonne.

La culture chinoise lui apprend une nouvelle simplicité de vie, antithèse de celle de $\mathrm{H}$. et, par conséquent, d’elle-même auparavant. L’existence humaine ne se questionne pas, elle s’impose tout simplement : «Nous sommes déjà arrivés à destination. Nous sommes arrivés en gare. Nous vivons » (p. 70). Et elle se voit confrontée à une culture où il n’y a pas de dieu pour veiller sur l'être humain, c'est à l'homme de le faire, de veiller sur lui et sur les autres personnes de son entourage qui lui sont importantes. Elle prend conscience du besoin de se prendre en main, d'agir sur les éléments de son avenir qu’elle doit maîtriser par elle-même. Dans cette conception de l'existence, règne l'idée de ‘fortune’ ou 'chance' : « La chance l'a quitté. On ne peut rien pour lui. Voilà. J'avais atterri dans un monde régi par la fortune et qui se fichait de mon bilan de bonté chrétienne»(p. 72). Elle se sent délivrée du poids de la religion, si imposante dans la société québécoise, et elle apprend à faire confiance à quelques collègues qui l'aident dans l'enquête policière qui l'obligera finalement à quitter la Chine. À travers ces personnages chinois de l'entourage et à travers la jeune fille du premier chapitre, elle va analyser et percevoir de manière plus favorable ses propres liens familiaux.

L’élément de l'eau est lié aussi au sexe et à la maternité. La narratrice se doute de la possibilité d’être enceinte et veut se procurer un test de grossesse à la pharmacie. Dans le chapitre neuf, elle reflète subtilement les préjugés de la société chinoise face à la sexualité. D’habitude, la narratrice communique bien en cantonais ou en mandarin avec l'employé, mais elle se heurte à l'incapacité d'être comprise quand il s’agit de quelle chose qui tient à une partie du corps féminin située " au sud de ma ceinture » (p. 84). Dans ce pays, elle est libérée du besoin de camoufler son absence de désir, elle se sent « seule avec Poissonne dans cet immense sanatorium » (p. 83). Elle révise sa vie amoureuse et devient consciente que la rencontre amoureuse avait toujours été pour elle une rencontre avec elle-même, jamais un acte de rencontre avec 'l'autre', «jamais ressenti de communion dans l'accouplement » (p. 86). Elle se voit libérée aussi bien du regard des hommes chinois toujours discrets que du regard des Blancs qu'elle côtoyait dans le travail ou dans le métro car ceux-ci voulaient se perdre dans des corps androgynes, sans âge et sans parole. Ils étaient eux aussi en fuite, la narratrice leur rappelait précisément ce qu’ils fuyaient. Cette délivrance va se traduire en une promesse intime de fonder sa famille sur un vrai acte d’amour alors que les sensations éprouvées sont de souplesse. Elle donne des qualités humaines à Poissonne qui devient son miroir de bonheur à cet instant-là. Elle est décrite en train de chanter au moment où elle lui apprend le résultat négatif du test de grossesse. Elle devint le reflet direct de la sensation de flottation, de sortir à la surface de l'eau car le fait d'être enceinte la renverrait à son passé.

A la fin de l'histoire, le lecteur perçoit le changement de personnalité, la narratrice se sent nourrie de soleils levants, car la ville de Hong Kong et Poissonne l'avaient 'ragaillardie', son esprit avait pris de l'aplomb et son corps du poids. Elle s'était renouvelée et emplie de la chaleur du Sud ; des images aquatiques deviennent, à la fin du livre, des images de lumière. Dans le camion qui la transporte à l'aéroport obligée d'abandonner le pays par la police, elle voit des dauphins dans la mer dont «les dos mouillés fulguraient sous le soleil nouveau » (p. 222). Elle est prête à partir pour le Canada remplie de force et son seul défi consisterait à savoir transporter avec elle à Montréal le sentiment de plénitude qu'elle ressentait en Orient.

\section{Conclusion}

Pour finir, on va essayer de faire apparaître de façon synthétique les thèmes qui se détachent principalement du roman HKPQ. D’abord, l'eau ouvre le récit associée à la partie négative de ce symbole parce qu'elle est liée à la mort ; mais nous avons déjà vu que sa disparition suppose une délivrance certaine pour elle. Cet événement cependant lui fait prendre conscience du besoin de s’éloigner pour se reconstruire à nouveau.

L'introduction ensuite d'un personnage un peu merveilleux comme celui de Poissonne révèle les thèmes de la régénération et de la métamorphose qui s’opèrent chez l'esprit et le corps de la narratrice. Elle va s’épanouir à la simple contemplation de la beauté de cet animal et la pousse à se battre pour qu'elle ne tombe pas dans les mains des malfaiteurs. D’ailleurs, à travers ce poisson-femelle, elle retrouve la foi en l'être humain et la joie de vivre. 
Enfin, l'eau est associée au thème de l'amitié avec son collègue chinois et sa famille qui lui offre aussi un modèle différent à celui que la narratrice connaissait, mais lui font aussi prendre distance de certaines valeurs religieuses qui conservent un certain poids dans la société québécoise ou, au moins, dans son imaginaire collectif.

\section{Références bibliographiques}

Aronsson, Mattias (2008). La thématique de l'eau dans l'œuvre de Marguerite Duras. Thèse de doctorat. Göteborgs: Acta Universitatis Gothoburgensis. Disponible à $\quad$ http://biblioteca.ucm.es/data/cont/media/www/pag61249/La\%20tem\%C3\%A1tica\%20del\%20agua.pdf> [Consulté le 12 décembre 2015]

Bachelard, Gaston (1942). L'eau et les rêves. Essai sur l'imagination de la matière. Paris : Librairie José Corti.

Bougerra, Larbi (2007). « Préface », dans Hana Aubry «, Imaginaire de l'eau, imaginaire du monde. Paris : La Dispute, pp. 9-28.

Brunel, Pierre (éd.) (1988) : Dictionnaire des mythes littéraires. Paris : Éditions du Rocher.

Dubois, Claude-Gilbert (1983). «Les réseaux symboliques et leur fonction littéraire », dans Versants : revue suisse des littératures romanes, $\mathrm{n}^{\circ}$ 4, p. 5-31. Disponible à < http://dx.doi.org/10.5169/seals-250735> ～[Cconsulté le 12 février 2016].

Chevalier, Jean et Alain Gheerbant (1995). Diccionario de los símbolos. Barcelona : Herder.

Goguey, Dominique et Fabien Dubouchet (2014). «Confrontation de l'imaginaire de l'eau dans l'épopée latine L'Énéide de Virgile et dans l'épopée celtique la Razzia des vache de Cooley ", dans Annales d’APLAES, pp.41-51.

Julien, Nadia (1989). Dictionnaire de symboles. Alleur (Belgique) : Marabout.

MoIR, Tristan-Frédéric. Dictionnaire des rêves. <http://tristan.moir.free.fr/dicoreve/symboleshtml/interpretation-des-revesdictionnaire.php?lettre=E\% $>$ [Consulté le 15 février 2016]

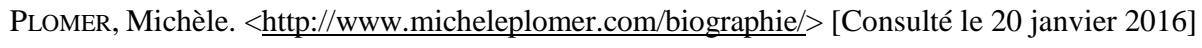

PLOMER, Michèle (2009). HKPQ. Montréal : Editions Marchand de feuilles.

SAXE FERnANDEZ, Eduardo (1979). «El agua y la muerte en Gastón Bachelard », Revista de filosofía de la universidad de Costa Rica, $\mathrm{n}^{\circ}$ 28, pp. 75-87.

TAROT, Camille (2007). «Existe-t-il une symbolique universelle de l'eau », dans Hana Aubry. Imaginaire de l'eau, imaginaire du monde. Paris : La Dispute, pp. 55-79. 